\title{
BRCA1 mutation in breast cancer patients: Analysis of prognostic factors and survival
}

\author{
JOANNA HUSZNO $^{1}$, ZOFIA KOŁOSZA ${ }^{2}$ and EWA GRZYBOWSKA ${ }^{3}$ \\ ${ }^{1}$ Outpatient Clinic; ${ }^{2}$ Biostatistics Unit; ${ }^{3}$ Center for Translational Research and Molecular Biology of Cancer, \\ Maria Skłodowska-Curie Memorial Cancer Center and Institute of Oncology, Gliwice Branch, 44-101 Gliwice, Poland
}

Received March 11, 2018; Accepted August 30, 2018

DOI: $10.3892 / \mathrm{ol} .2018 .9770$

\begin{abstract}
The presence of BRCA1 mutations is associated with an increased risk of breast and ovarian cancer. The present study compared clinicopathological characteristics and overall survival (OS) of hereditary and sporadic breast cancer. Using data collected from a previous study conducted between 2007-2016 at the Maria Skłodowska Curie Cancer Center and Institute of Oncology (Gliwice, Poland), the prognostic factors and survival in 60 breast cancer mutation carriers were analyzed. A control group was selected from the breast cancer patients without BRCA mutations $(n=386)$. BRCA mutation carriers had significantly worse survival when compared with non-carriers $(\mathrm{P}=0.017)$. The 10-year OS rate was $78.0 \%$ for all analyzed groups: $65.9 \%$ for BRCA mutation carriers and $81.1 \%$ for non-carriers. In the univariate analyses, BRCA mutation carriers had a significantly higher risk of mortality in comparison to non-carriers [hazard ratio $(\mathrm{HR})=1.87 ; 95 \%$ confidence interval $(\mathrm{CI})$ 1.08-3.25]. Increased tumor size $(\mathrm{HR}=3.64)$, lymph node metastases $(\mathrm{HR}=2.45)$ and higher histological grade $(\mathrm{HR}=2.84)$ were significant factors for worse OS. Positive estrogen receptor status was associated with a better $\mathrm{OS}(\mathrm{HR}=0.49, \mathrm{P}=0.022)$. Age $\leq 40$ years $(\mathrm{HR}=0.48, \mathrm{P}=0.081)$ was an insignificantly favorable factor. The 10-year survival rate was significantly decreased in patients with BRCA1 mutation. Therefore, negative factors for OS in mutation carriers included lymph nodes metastases, negative steroid receptor status and increased tumor size.
\end{abstract}

\section{Introduction}

The presence of $B R C A$ mutations increases the risk of breast $(\sim 80 \%)$ and ovarian cancer $(\sim 40 \%)$. The incidence of $B R C A$

Correspondence to: Dr Joanna Huszno, Outpatient Clinic, Maria Skłodowska-Curie Memorial Cancer Center and Institute of Oncology, Gliwice Branch, ul. Wybrzeze Armii Krajowej 15, 44-101 Gliwice, Poland

E-mail: joahus@wp.pl

Key words: breast cancer, BRCA1-mutation, prognostic factor, Silesian population mutations in breast and ovarian cancer are $<1-7 \%$ for $B R C A 1$ and $1-3 \%$ for $B R C A 2$ independently from family history or age at diagnosis. In literature, a family history of breast or ovarian cancer, young age at diagnosis, male breast cancer or multiple tumors (bilateral breast cancer or breast and ovarian cancer in the same patient) occur more often in BRCA mutation carriers. The median time of diagnosis of breast cancer in patients with germline $B R C A$ mutation is lower (in age under 50 years) than for patients with sporadic cancer (1). External factors which can modify $B R C A$ associated breast cancer risk are hormonal and reproductive factors such as pregnancy, history of breast feeding and oral contraceptives $(2,3)$.

It has previously been demonstrated that tumors in patients with $B R C A 1$ mutation frequently exhibit negative steroid receptor status, with expression of p53 protein. Mutations in TP53 gene also seem to be increased in tumors with BRCA1 mutation. A previous study indicated that familial breast cancers with $B R C A 1$ mutation are different from $B R C A 2$ tumors and sporadic cancers (4).

The triple negative breast cancer (TNBC) phenotype is the most commonly observed molecular subtype in patients with $B R C A 1$ mutation. The presence of triple negative diseases in $B R C A 1$ mutation carriers is higher than in sporadic breast cancer patients and is $11-20 \%$ (5). Recent data show that survival rate of $B R C A$ carriers who were administrated systemic treatment (chemotherapy) was similar to non-carriers $(6,7)$. Various studies both clinical and preclinical, showed that $B R C A$ is an important factor affecting chemotherapy response and treatment toxicity in breast cancer patients (8). In Poland, three founder mutations in BRCA1 (i.e., 5382insC, C61G, 4153delA) are under investigation (9).

In the present study, we compare hereditary and sporadic breast cancer according to clinicopathological factors and overall survival (OS) time.

\section{Materials and methods}

In a study conducted in the years 2007-2016 in the Maria Skłodowska Curie Memorial Cancer Center and Institute of Oncology (COI; Gliwice, Poland), we analyzed prognostic factors and survival in 60 patients with breast cancer with confirmed BRCA1 mutations. A control group was selected from breast cancer patients without the $B R C A$ mutation $(\mathrm{n}=386)$. The patients in both groups were treated according to 
the same protocol. All patients had signed a written informed consent allowing their biological material to be used in clinical research.

All patients were females diagnosed, treated and followed up at the COI in Gliwice. Patients underwent clinical follow-up examinations every three months in the first two years, every six months afterwards until the fifth year after diagnosis and every year subsequently. Inclusion criteria were: Breast cancer confirmed by microscopic examination, performance status ZUBROD 0-1, age above 18, the correct value of renal and liver function and normal values of bone marrow. The data of age at onset, menopausal status, surgical procedure, disease stage according to TNM classification, histology, estrogen and progesterone receptor (PR) status, HER2 status and contralateral breast cancer were gathered from hospital records and pathology reports. The analysis of patient medical records was performed according to national law regulation.

All patients had genetic tests and consultation in Genetic Outpatient Clinic. Mutation profile was assessed by RFLP-PCR technique. We evaluated the three most common mutations in the Polish population, including 5382insC, C61G and 4153delA. All patients were tested for the presence of $B R C A 1$ and $B R C A 2$ mutations. Mutation analysis was conducted by a multiplex allele-specific polymerase chain reaction assay.

Statistical analysis was carried out using STATISTICA 7 software (StatSoft, Inc., Tulsa, OK, USA). The frequency of side effects was monitored. The qualitative features were presented as the percentage of their occurrence and evaluated with Fisher's test and $\chi^{2}$ test with Yates correction. $\mathrm{P}<0.05$ was considered to indicate a statistically significant difference. Prognostic factors of OS were estimated by Cox proportional hazards model. The probability of survival was estimated using the Kaplan-Meier method.

\section{Results}

Patient characteristics. For the total group of 446 cases, the median age at diagnosis was 51.8 years (range, 23.7-78.3 years). In $B R C A$ mutation carriers $(n=60)$ and non-carriers $(n=386)$ the median age was 43.5 years (range, 23.7-74.4 years) and 53.1 years (range, 25.6-78.3 years), respectively. $B R C A$ carriers were significantly younger $(\mathrm{P}<0.0001)$ than non-carriers. A total of 263 women $(59.0 \%)$ were in premenopausal period ( $80 \%$ carriers and $56 \%$ non-carriers) $(\mathrm{P}=0.0004)$. The majority of patients had early stage breast cancer. Distant metastases were observed only in $7(1.6 \%)$ of women (1 case in $B R C A$ mutation carriers and 6 in non-carriers). Lymph node metastases $(\mathrm{N}+)$ was detected more frequently in non-carriers (45.9\%; vs. $18.3 \%, \mathrm{P}=0.0001$ ). Conversely, locally advanced breast cancer (T3-T4) was reported frequently in BRCA mutation carriers $(38.3 \%$ vs. $19.4 \%, \mathrm{P}=0.002)$. Lobular invasive carcinoma was reported more often in patients without $B R C A$ mutation than in BRCA carriers (12.2\% vs. $5 \%$ ). As expected, patients with $B R C A$ mutation had more frequent estrogen receptor (ER; $66.7 \%$ vs. $35.5 \%, \mathrm{P}=0.0001)$ and PR (71.7\% vs. $41.7 \%, \mathrm{P}=0.0002)$ negative receptor status, higher histological grade (G3; $50 \%$ vs. $29.5 \%, \mathrm{P}=0.002)$, negative HER2 receptor status $(98.3 \%$ vs. $56.2 \%, \mathrm{P}=0.0001)$ and TNBC $(61.7 \%$ vs. $15.0 \%, \mathrm{P}=0.0001)$. There was also an observed predisposition to the development of secondary cancers in mutation carriers (35\% vs. 9.6\%, $\mathrm{P}=0.0001)$. Clinicopathological patient characteristics are presented in Table I.

In the subgroup analysis, there were no significant differences between younger ( $\leq 40$ years) and older ( $>40$ years) $B R C A$ mutation carriers according to clinicopathological factors. Among younger patients ( $\leq 40$ years) there was an observed increased occurrence of TNBC (68\% vs. 58\%; $\mathrm{P}=0.583)$, tumors with negative ER status (ER-) (77\% vs. $60 \% ; \mathrm{P}=0.258)$ and with negative PR status (PR-) (77\% vs. $68 \% ; \mathrm{P}=0.560)$ and without HER2 overexpression (100\% vs. 97.4\%, $\mathrm{P}=1.00)$ (Table II). In $B R C A$ non-carriers, younger patients ( $\leq 40$ years) in comparison to older exhibited an increased rate of diagnosis of TNBC (20.0\% vs. $14.4 \%$, $\mathrm{P}=0.373)$, tumors with ER- status $(42.2 \%$ vs. $34.6 \%, \mathrm{P}=0.324)$ and HER2 overexpression ( $48.9 \%$ vs. $43.1 \%, \mathrm{P}=0.524)$. There were no differences observed in negative PR status (PR-) $(42.2 \%$ vs. $41.6 \%)$.

Treatment strategies. Treatment strategies are presented in Table III. The surgical treatment was performed in 402 (90.1\%) patients, including mastectomy for $292(65.5 \%)$ and breast conserving treatment (BCT) for 110 (24.7\%). BCT was conducted more often in non-carriers in comparison to carriers $(28.2 \%$ vs. $21.6 \%, \mathrm{P}=0.401)$. Radiotherapy was administered to $66.7 \%$ of mutation carriers and $67.1 \%$ non-carriers $(\mathrm{P}=1.00)$. The total radiotherapy dose administered was $50 \mathrm{~Gy}$ in 25 fractions. If indicated, a boost was delivered. All patients underwent chemotherapy. A total of $97.3 \%$ (434) patients received anthracycline based chemotherapy (AC, FAC) at The Clinical and Experimental Oncology Department. Chemotherapy regiments with taxanes (paclitaxel) were used in $13 \%$ of patients. Patients with steroid positive receptor breast cancer were treated with anti-estrogen therapy: $61.1 \%$ of non-carriers and $30.0 \%$ of $B R C A$ mutation carriers $(\mathrm{P}<0.0001)$. The lower frequency of HT in carriers was due to the high frequency of ER (-) in that group. Trastuzumab was used in women with HER 2 positive breast cancer confirmed by immunohistochemistry examination or by the FISH method (gene amplification) (1.7\% BRCA carriers and $41.2 \%$ non-carriers, $\mathrm{P}<0.0001)$.

Survival analysis in BRCA (-) negative patients. Patients with positive nodes $(\mathrm{N}+)$ exhibited a significantly worse OS than those without node involvement (5-year survival rate $82 \%$ vs. $93 \%, \mathrm{P}=0.0008$ ) (Fig. 1). Risk of mortality was 2.7 fold higher for patients with lymph node metastases. The 5 year OS rate depending on the depth (T) was $97 \%$ for $\mathrm{T} 1,88 \%$ for $\mathrm{T} 2$ and $74 \%$ for the T3-T4 (Fig. 2). The risk of mortality depended on the stage of the disease and was higher at the advanced T3-T4 stages, $\mathrm{HR}=4.7 ;(\mathrm{P}=0.0006)$. Patients with positive ER status (ER+) had a longer OS rate (5-year OS $91 \%$ vs. $82 \%, \mathrm{P}=0.054$ ) however this was not significant (Fig. 3). Patients with tumor HER2 overexpression had a lower OS rate (5-year OS $86 \%$ vs. 89\%, $\mathrm{P}=0.273$ ) (Fig. 4), which was also not significant. Younger patients ( $\leq 40$ years) had an increased OS rate (5-year OS 93\% vs. 87\%; $\mathrm{P}=0.167$ ) (Fig. 5) however this was again not significant. They also had a lower risk of mortality $(\mathrm{HR}=0.36 ; \mathrm{P}=0.167)$ compared with 
Table I. Clinicopathological patient's characteristics according to $B R C A l$ mutation carriers.

\begin{tabular}{|c|c|c|c|c|c|c|c|}
\hline \multirow[b]{2}{*}{ Factors } & \multirow[b]{2}{*}{$\mathrm{n}$} & \multirow{2}{*}{$\begin{array}{l}\text { Percentage of } \\
\text { total } \mathrm{n}(\%)\end{array}$} & \multicolumn{2}{|c|}{$B R C A l$ carriers } & \multicolumn{2}{|c|}{$B R C A 1$ non carriers } & \multirow[b]{2}{*}{ P-value } \\
\hline & & & $\mathrm{n}$ & $\%$ of $n$ & $\mathrm{n}$ & $\%$ of $n$ & \\
\hline Total cases & 446 & 100 & 60 & 100 & 386 & 100 & - \\
\hline \multicolumn{8}{|c|}{$\begin{array}{l}\text { Age (range, } 24-78 \text { years; } \\
\text { median } 52 \text { years) }\end{array}$} \\
\hline$\leq 65$ & 386 & 86.5 & 55 & 91.7 & 331 & 85.8 & \multirow[t]{2}{*}{0.308} \\
\hline$>65$ & 60 & 13.5 & 5 & 8.3 & 55 & 14.2 & \\
\hline \multicolumn{8}{|l|}{ Age (years) } \\
\hline$\leq 40$ & 67 & 15.0 & 22 & 36.7 & 45 & 11.7 & \multirow[t]{2}{*}{0.0001} \\
\hline$>40$ & 379 & 85.0 & 38 & 63.3 & 341 & 88.3 & \\
\hline \multicolumn{8}{|l|}{ Menopausal status } \\
\hline Postmenopausal & 183 & 41.0 & 12 & 20.0 & 171 & 44.3 & \multirow[t]{2}{*}{0.0004} \\
\hline Premenopausal & 263 & 59.0 & 48 & 80.0 & 215 & 55.7 & \\
\hline \multicolumn{8}{|l|}{ Clinical staging } \\
\hline I & 90 & 20.2 & 8 & 13.3 & 82 & 21.2 & \multirow[t]{7}{*}{0.030} \\
\hline IIA & 136 & 30.5 & 23 & 38.3 & 113 & 29.3 & \\
\hline IIB & 128 & 28.7 & 23 & 38.3 & 105 & 27.2 & \\
\hline IIIA & 69 & 15.5 & 2 & 3.3 & 67 & 17.4 & \\
\hline IIIB & 11 & 2.5 & 3 & 5.0 & 8 & 2.1 & \\
\hline IIIC & 5 & 1.1 & 0 & 0.0 & 5 & 1.3 & \\
\hline IV & 7 & 1.6 & 1 & 1.7 & 6 & 1.6 & \\
\hline \multicolumn{8}{|l|}{$\mathrm{T}$} \\
\hline $\mathrm{T} 1$ & 131 & 29.4 & 10 & 16.7 & 121 & 31.3 & \multirow[t]{4}{*}{0.0001} \\
\hline $\mathrm{T} 2$ & 217 & 48.7 & 27 & 45.0 & 190 & 49.2 & \\
\hline $\mathrm{T} 3$ & 77 & 17.3 & 14 & 23.3 & 63 & 16.3 & \\
\hline $\mathrm{T} 4$ & 21 & 4.7 & 9 & 15.0 & 12 & 3.1 & \\
\hline \multicolumn{8}{|c|}{ Clinical staging nodes } \\
\hline No & 258 & 57.8 & 49 & 81.7 & 209 & 54.1 & \multirow[t]{4}{*}{0.001} \\
\hline N1 & 133 & 29.8 & 8 & 13.3 & 125 & 32.4 & \\
\hline $\mathrm{N} 2$ & 47 & 10.5 & 3 & 5.0 & 44 & 11.4 & \\
\hline N3 & 8 & 1.8 & 0 & 0.0 & 8 & 2.1 & \\
\hline \multicolumn{8}{|l|}{ G } \\
\hline G1 & 27 & 6.1 & 1 & 1.7 & 26 & 6.7 & \multirow[t]{4}{*}{0.002} \\
\hline $\mathrm{G} 2$ & 111 & 24.9 & 6 & 10.0 & 105 & 27.2 & \\
\hline G3 & 144 & 32.3 & 30 & 50.0 & 114 & 29.5 & \\
\hline Missing & 164 & 36.8 & 23 & 38.3 & 141 & 36.5 & \\
\hline \multicolumn{8}{|l|}{ Tumor type } \\
\hline Ductal invasive & 363 & 81.4 & 56 & 93.3 & 307 & 79.5 & 0.035 \\
\hline Lobular invasive & 50 & 11.2 & 3 & 5.0 & 47 & 12.2 & \\
\hline Other & 33 & 7.4 & 1 & 1.7 & 32 & 8.3 & \\
\hline ER & & & & & & & \\
\hline Negative & 177 & 39.7 & 40 & 66.7 & 137 & 35.5 & 0.0001 \\
\hline Positive & 269 & 60.3 & 20 & 33.3 & 249 & 64.5 & \\
\hline PR & & & & & & & \\
\hline Negative & 204 & 45.7 & 43 & 71.7 & 161 & 41.7 & 0.0002 \\
\hline Positive & 242 & 54.3 & 17 & 28.3 & 225 & 58.3 & \\
\hline Steroid receptor & & & & & & & \\
\hline Negative & 161 & 36.1 & 37 & 61.7 & 124 & 32.1 & 0.0002 \\
\hline Positive & 285 & 63.9 & 23 & 38.3 & 262 & 67.9 & \\
\hline
\end{tabular}


Table I. Continued.

\begin{tabular}{|c|c|c|c|c|c|c|c|}
\hline \multirow[b]{2}{*}{ Factors } & \multirow[b]{2}{*}{$\mathrm{n}$} & \multirow{2}{*}{$\begin{array}{l}\text { Percentage of } \\
\text { total } \mathrm{n}(\%)\end{array}$} & \multicolumn{2}{|c|}{$B R C A 1$ carriers } & \multicolumn{2}{|c|}{$B R C A 1$ non carriers } & \multirow[b]{2}{*}{ P-value } \\
\hline & & & $\mathrm{n}$ & $\%$ of $\mathrm{n}$ & $\mathrm{n}$ & $\%$ of $\mathrm{n}$ & \\
\hline \multicolumn{8}{|c|}{ HER 2 overexpression } \\
\hline Negative & 276 & 61.9 & 59 & 98.3 & 217 & 56.2 & 0.0001 \\
\hline Positive & 170 & 38.1 & 1 & 1.7 & 169 & 43.8 & \\
\hline \multicolumn{8}{|c|}{ Triple negative } \\
\hline No & 351 & 78.7 & 23 & 38.3 & 328 & 85.0 & 0.0001 \\
\hline Yes & 95 & 21.3 & 37 & 61.7 & 58 & 15.0 & \\
\hline
\end{tabular}

T, tumor size; N, node; ER, estrogen receptor; HER2, human epidermal growth factor receptor 2; PR, progesterone receptor; G, grade.

Table II. Patient's characteristics according to age.

\begin{tabular}{|c|c|c|c|c|c|c|c|}
\hline \multirow[b]{2}{*}{ Factors } & \multirow[b]{2}{*}{ Total $n$} & \multirow[b]{2}{*}{ Percentage of total n (\%) } & \multicolumn{2}{|c|}{ Age $\leq 40$ years } & \multicolumn{2}{|c|}{ Age $>40$ years } & \multirow[b]{2}{*}{ P-value } \\
\hline & & & $\mathrm{n}$ & $\%$ of $n$ & $\mathrm{n}$ & $\%$ of $n$ & \\
\hline$B R C A 1$ carriers & 60 & 100 & 22 & 100 & 38 & 100 & - \\
\hline \multicolumn{8}{|l|}{$\mathrm{T}$} \\
\hline $\mathrm{T} 1$ & 10 & 16.7 & 4 & 18.2 & 6 & 15.8 & 0.635 \\
\hline $\mathrm{T} 2$ & 27 & 45.0 & 8 & 36.4 & 19 & 50.0 & \\
\hline T3-T4 & 23 & 38.3 & 10 & 45.5 & 13 & 34.2 & \\
\hline \multicolumn{8}{|c|}{ Clinical staging nodes } \\
\hline N0 & 49 & 81.7 & 20 & 90.9 & 29 & 76.3 & 0.0001 \\
\hline $\mathrm{N}+$ & 11 & 18.3 & 2 & 9.1 & 9 & 23.7 & \\
\hline \multicolumn{8}{|l|}{ G } \\
\hline G1-G2 & 7 & 11.7 & 1 & 4.5 & 6 & 15.8 & 0.261 \\
\hline G3 & 30 & 50.0 & 10 & 45.5 & 20 & 52.6 & \\
\hline Missing & 23 & 38.3 & 11 & 50.0 & 12 & 31.6 & \\
\hline \multicolumn{8}{|l|}{$\mathrm{ER}$} \\
\hline Negative & 40 & 66.7 & 17 & 77.3 & 23 & 60.5 & 0.258 \\
\hline Positive & 20 & 33.3 & 5 & 22.7 & 15 & 39.5 & \\
\hline \multicolumn{8}{|l|}{ PR } \\
\hline Negative & 43 & 71.7 & 17 & 77.3 & 26 & 68.4 & 0.560 \\
\hline Positive & 17 & 28.3 & 5 & 22.7 & 12 & 31.6 & \\
\hline \multicolumn{8}{|c|}{ HER 2 overexpression } \\
\hline Negative & 59 & 98.3 & 22 & 100.0 & 37 & 97.4 & 1.00 \\
\hline Positive & 1 & 1.7 & 0 & 0.0 & 1 & 2.6 & \\
\hline \multicolumn{8}{|l|}{ Triple negative } \\
\hline No & 23 & 38.3 & 7 & 31.8 & 16 & 42.1 & 0.583 \\
\hline Yes & 37 & 61.7 & 15 & 68.2 & 22 & 57.9 & \\
\hline
\end{tabular}

T, tumor size; N, node; ER, estrogen receptor; HER2, human epidermal growth factor receptor 2; PR, progesterone receptor; G, grade.

older patients. In uni- and multivariate analyses, increased tumor size, lymph node metastasis and higher tumor grade were all associated with increased risk of mortality (Table IV). Similarly, steroid receptor status (ER negative) insignificantly increased risk of mortality.
Survival analysis in BRCA (+) mutation carriers. The 5-year OS rate was 77.3\% [95\% confidence interval (CI), 66.4-88.2\%]. Patients with lymph node metastases $(\mathrm{N}+)$ had a significantly lower 5-year OS compared with patients without lymph node involvement $(52 \%$ vs. $83 \%, \mathrm{P}=0.03)$ and 3.0 fold higher risk of 
Table III. Treatment strategy according to BRCAl mutation.

\begin{tabular}{|c|c|c|c|c|c|c|c|}
\hline \multirow[b]{2}{*}{ Treatment } & \multirow[b]{2}{*}{ Total n } & \multirow[b]{2}{*}{ Percentage of total $\mathrm{n}(\%)$} & \multicolumn{2}{|c|}{$B R C A l$ carriers } & \multicolumn{2}{|c|}{$\begin{array}{c}\text { BRCAl } \\
\text { non carriers }\end{array}$} & \multirow[b]{2}{*}{ P-value } \\
\hline & & & $\mathrm{n}$ & $\%$ of $n$ & $\mathrm{n}$ & $\%$ of $n$ & \\
\hline Total cases & 446 & 100 & 60 & 100 & 386 & 100 & - \\
\hline \multicolumn{8}{|l|}{ Chemotherapy regimen } \\
\hline AC FAC & 376 & 84.3 & 44 & 73.3 & 332 & 86.0 & 0.005 \\
\hline $\mathrm{AC}+$ taxanes & 58 & 13.0 & 11 & 18.3 & 47 & 12.2 & \\
\hline $\mathrm{CMF}$ & 12 & 2.7 & 5 & 8.3 & 7 & 1.8 & \\
\hline \multicolumn{8}{|l|}{ Trastuzumab therapy } \\
\hline Yes & 160 & 35.9 & 1 & 1.7 & 159 & 41.2 & 0.0001 \\
\hline No & 286 & 64.1 & 59 & 98.3 & 227 & 58.8 & \\
\hline \multicolumn{8}{|l|}{ Hormonotherapy } \\
\hline Yes & 254 & 57.0 & 18 & 30.0 & 236 & 61.1 & 0.0001 \\
\hline No & 192 & 43.0 & 42 & 70.0 & 150 & 38.9 & \\
\hline \multicolumn{8}{|l|}{ Local treatment } \\
\hline Mastectomy & 292 & 65.5 & 40 & 66.7 & 252 & 65.3 & 0.224 \\
\hline Breast conservation surgery & 110 & 24.7 & 11 & 18.3 & 99 & 25.6 & \\
\hline Without surgery & 44 & 9.9 & 9 & 15.0 & 35 & 9.1 & \\
\hline \multicolumn{8}{|l|}{ Radiotherapy } \\
\hline Yes & 299 & 67.0 & 40 & 66.7 & 259 & 67.1 & 1.00 \\
\hline No & 147 & 33.0 & 20 & 33.3 & 127 & 32.9 & \\
\hline
\end{tabular}

AC, Adriamycin (or doxorubicin; $60 \mathrm{mg} / \mathrm{m}^{2}$ ) and Cyclophosphamide $\left(600 \mathrm{mg} / \mathrm{m}^{2}\right)$ treatment; FAC, Fluorouracil $\left(500 \mathrm{mg} / \mathrm{m}^{2}\right)$, Adriamycin (or doxorubicin; $50 \mathrm{mg} / \mathrm{m}^{2}$ ) and Cyclophosphamide $\left(500 \mathrm{mg} / \mathrm{m}^{2}\right)$ treatment; CMF, Cyclophosphamide $\left(100 \mathrm{mg} / \mathrm{m}^{2}\right)$, Methotrexate $\left(40 \mathrm{mg} / \mathrm{m}^{2}\right)$ and Fluorouracil $\left(600 \mathrm{mg} / \mathrm{m}^{2}\right)$ treatment.

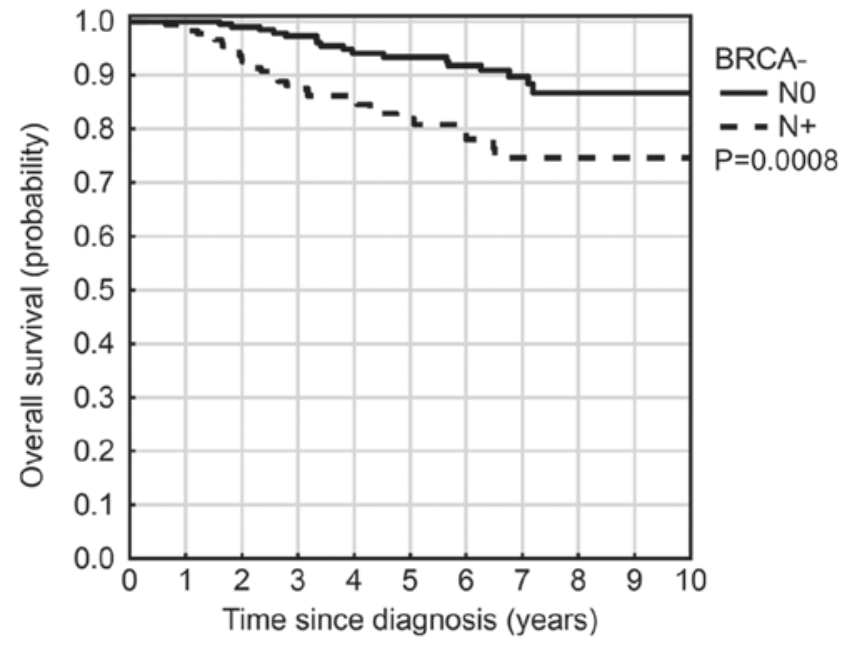

Figure 1. Overall survival analysis in BRCA(-) negative patients according to lymph node involvement. $\mathrm{P}=0.0008$. $\mathrm{N}$, node.

death (Fig. 6). 5-year OS was associated with tumor size (T) and was $90 \%$ for T1, $84 \%$ for T2 and $63 \%$ for T3-T4. The risk of mortality depended on stage of disease and was the greatest at the advanced T3-T4 stages, HR=5.07; (95\% CI, 0.64-40.33 $\mathrm{P}=0.125$ ) (Fig. 7). Patients who had tumors with ER+ status had an insignificantly higher 5-year OS (83\% vs. 74\%, $\mathrm{P}=0.417$ ) (Fig. 8). Younger patients ( $\leq 40$ years) exhibited an

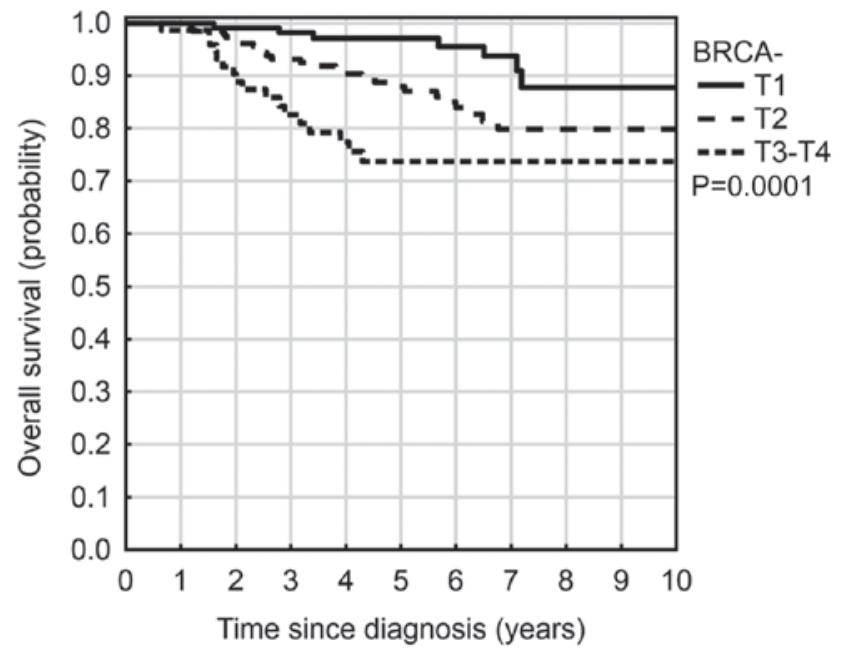

Figure 2. Overall survival analysis in BRCA(-) negative patients according to tumor size. $\mathrm{P}=0.0001$. $\mathrm{T}$, tumor size.

insignificantly higher OS ( $82 \%$ vs. $75 \%$; $\mathrm{P}=0.310)$ (Table IV). In univariate analysis, lymph node metastasis was a significant prognostic factor. In multivariate analysis, lymph node metastases $(\mathrm{HR}=3.29, \mathrm{P}=0.036)$ and $\mathrm{ER}$ - status $(\mathrm{HR}=7.14, \mathrm{P}=0.049)$ were identified as negative prognostic factors in $B R C A$ mutation carriers. Conversely, TNBC was a favorable prognostic factor in this group $(\mathrm{HR}=0.20, \mathrm{P}=0.073$ ) (Table IV). 


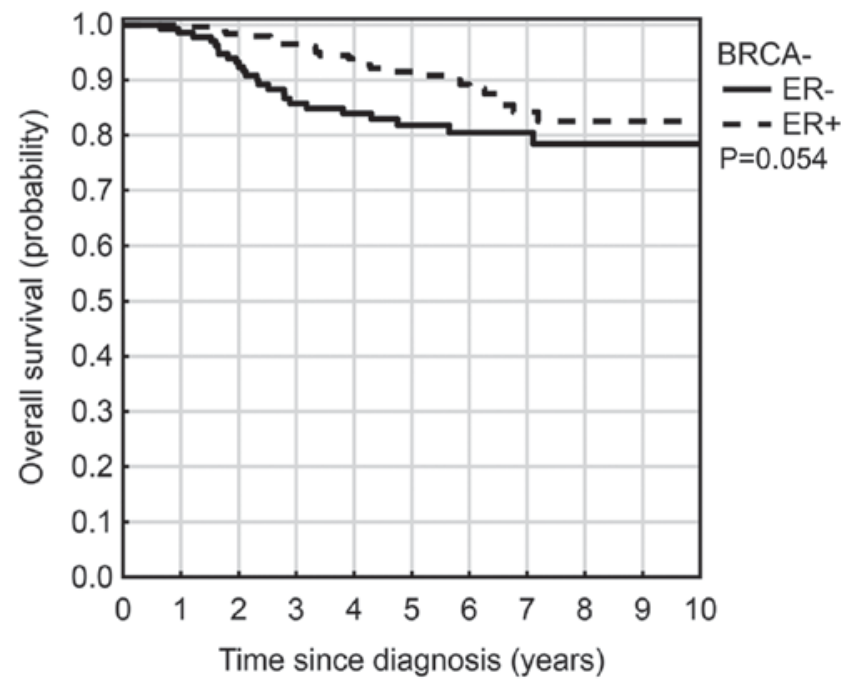

Figure 3. Overall survival analysis in BRCA(-) negative patients according to steroid receptor status. $\mathrm{P}=0.054$. ER, estrogen receptor.

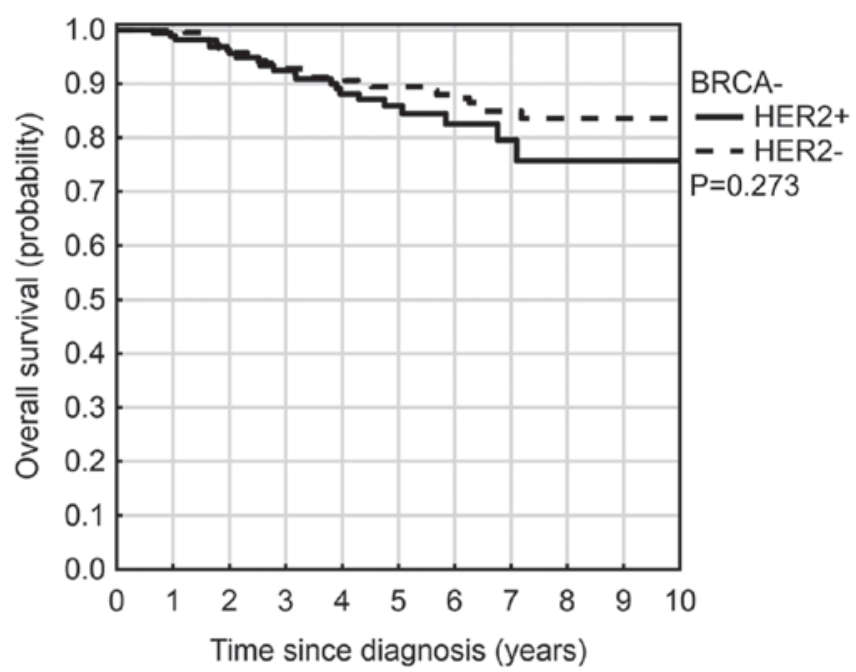

Figure 4. Overall survival analysis in BRCA(-) negative patients according to HER2 overexpression. $\mathrm{P}=0.273$. HER2, human epidermal growth factor receptor 2 .

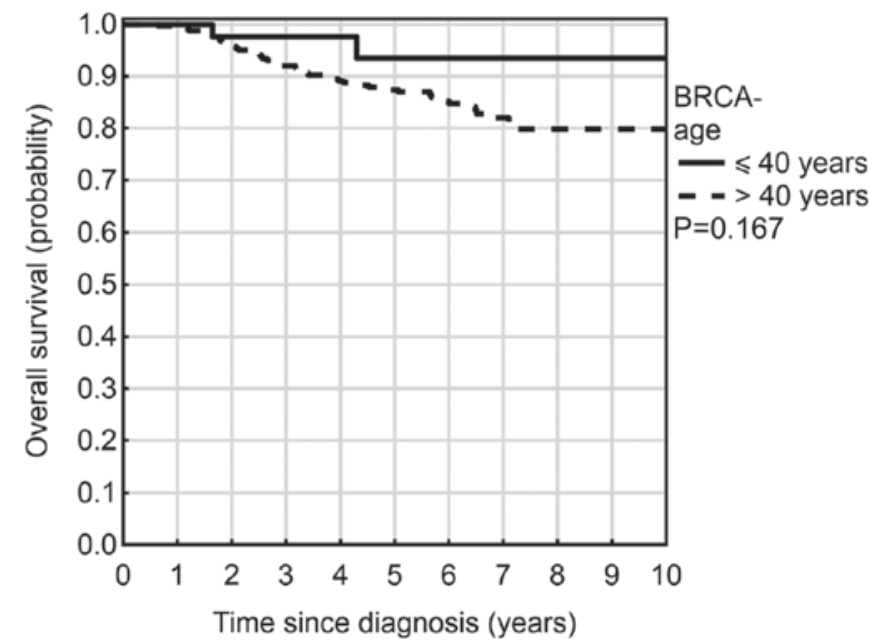

Figure 5. Overall survival analysis in BRCA(-) negative patients according to patients age. $\mathrm{P}=0.273$.

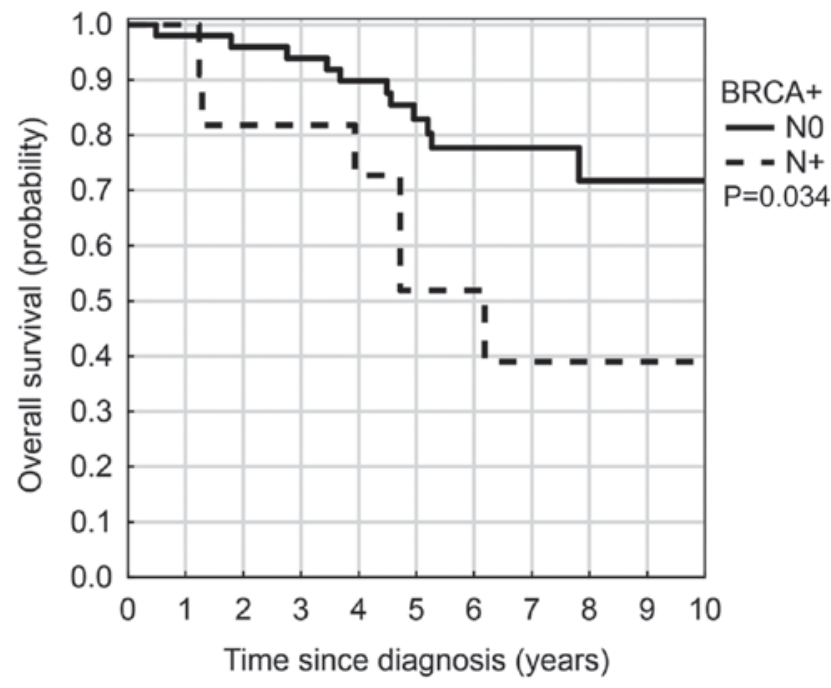

Figure 6. Overall survival analysis in $\mathrm{BRCA}(+)$ positive patients according to lymph node involvement. $\mathrm{P}=0.034$. $\mathrm{N}$, node.

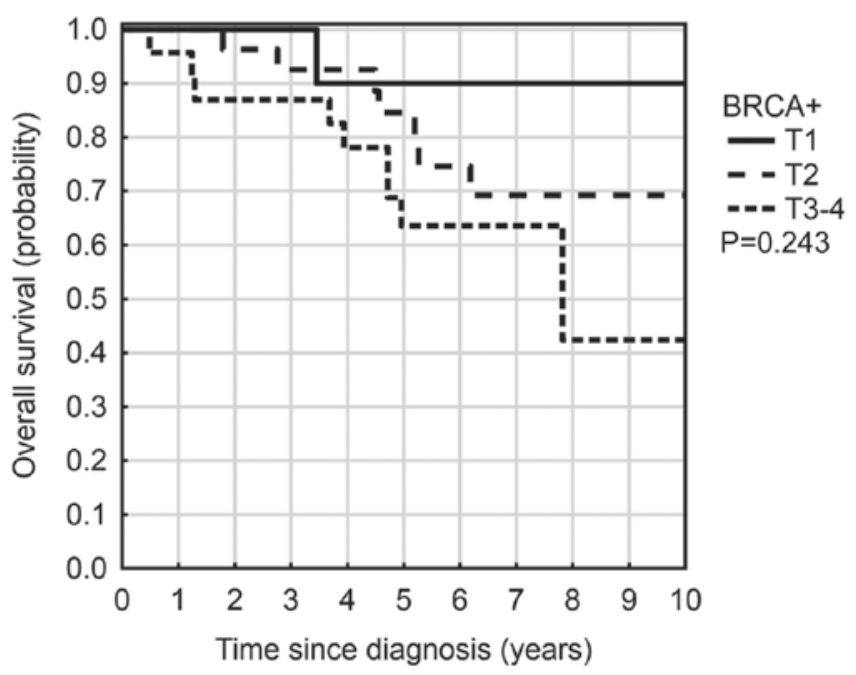

Figure 7. Overall survival analysis in $\mathrm{BRCA}(+)$ positive patients according to tumor. $\mathrm{P}=0.243$. $\mathrm{T}$, tumor size.

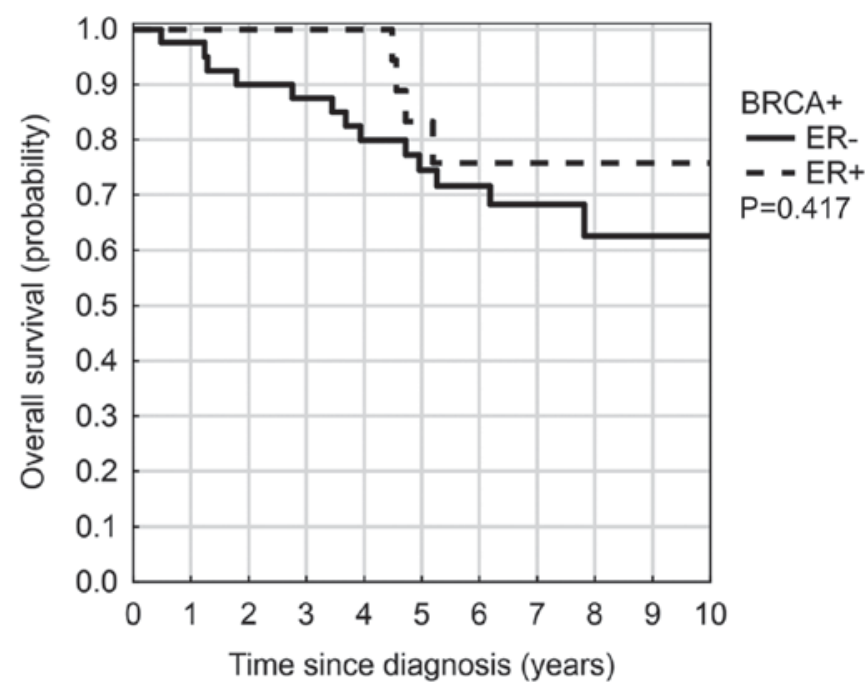

Figure 8. Overall survival analysis in $\mathrm{BRCA}(+)$ positive patients according to steroid receptor status. $\mathrm{P}=0.417$. $\mathrm{ER}$, estrogen receptor. 
Table IV. 5-year survival rates, and uni- and multivariate hazard ratios for mortalities in BRCAl non-carriers and carriers.

A, BRCAl non-carriers

\begin{tabular}{|c|c|c|c|c|c|c|c|c|}
\hline \multirow[b]{2}{*}{ Factor } & \multirow[b]{2}{*}{ Total $n$} & \multirow{2}{*}{$\begin{array}{l}5 \text {-year survival } \\
\text { rate }(\%)\end{array}$} & \multirow{2}{*}{$\begin{array}{l}\text { Test log rank } \\
\text { P-value }\end{array}$} & \multicolumn{2}{|c|}{ Univariate } & \multicolumn{3}{|c|}{ Multivariate } \\
\hline & & & & HR & $\mathrm{P}$-value & HR & $95 \% \mathrm{CI}$ & P-value \\
\hline Total cases & 386 & 88.1 & - & - & - & - & - & - \\
\hline \multicolumn{9}{|l|}{ Age (years) } \\
\hline$\leq 40$ & 45 & 93.5 & & 0.36 & 0.161 & 0.37 & $0.09-1.53$ & 0.169 \\
\hline$>40$ & 341 & 87.4 & 0.167 & 1.0 & & 1.0 & & \\
\hline \multicolumn{9}{|l|}{ T Stage } \\
\hline $\mathrm{T} 1$ & 121 & 97.1 & & 1.0 & & 1.0 & & \\
\hline $\mathrm{T} 2$ & 190 & 87.9 & 0.0001 & 2.59 & 0.026 & 2.26 & $0.98-5.22$ & 0.057 \\
\hline $\mathrm{T} 3-\mathrm{T} 4$ & 75 & 73.7 & & 4.71 & 0.0006 & 3.32 & $1.34-8.20$ & 0.009 \\
\hline \multicolumn{9}{|c|}{ Clinical staging nodes } \\
\hline No & 209 & 93.4 & & 1.0 & & 1.0 & & \\
\hline $\mathrm{N}+$ & 177 & 81.9 & 0.0008 & 2.67 & 0.001 & 2.40 & $1.30-4.42$ & 0.005 \\
\hline \multicolumn{9}{|l|}{ G } \\
\hline G1-G2 & 131 & 94.8 & & 1.0 & & 1.0 & & \\
\hline G3 & 114 & 84.0 & 0.0039 & 3.71 & 0.004 & 2.93 & $1.19-7.19$ & 0.019 \\
\hline Missing & 141 & 85.3 & & 3.04 & 0.009 & 2.95 & $1.27-6.86$ & 0.012 \\
\hline \multicolumn{9}{|l|}{ ER status } \\
\hline Negative & 137 & 81.8 & & 1.0 & & 1.0 & & \\
\hline Positive & 249 & 91.5 & 0.054 & 0.58 & 0.057 & 0.54 & $0.28-1.04$ & 0.064 \\
\hline \multicolumn{9}{|c|}{ Triple negative } \\
\hline No & 328 & 88.5 & & 1.0 & & 1.0 & & \\
\hline Yes & 58 & 85.2 & 0.745 & 1.12 & 0.754 & 0.69 & $0.30-1.59$ & 0.382 \\
\hline
\end{tabular}

B, BRCAl carriers

\begin{tabular}{|c|c|c|c|c|c|c|c|c|}
\hline \multirow[b]{2}{*}{ Factor } & \multirow[b]{2}{*}{$\mathrm{N}$} & \multirow{2}{*}{$\begin{array}{l}5 \text {-year survival } \\
\text { rate }\end{array}$} & \multirow{2}{*}{$\begin{array}{l}\text { Test log rank } \\
\text { P-value }\end{array}$} & \multicolumn{2}{|c|}{ Univariate } & \multicolumn{3}{|c|}{ Multivariate } \\
\hline & & & & HR & P-value & HR & $95 \%$ CI & P-value \\
\hline Total cases & 60 & 77.3 & - & - & - & - & - & - \\
\hline \multicolumn{9}{|l|}{ Age (years) } \\
\hline$\leq 40$ & 22 & 81.8 & 0.310 & 0.59 & 0.326 & 0.44 & $0.12-1.60$ & 0.213 \\
\hline$>40$ & 38 & 75.0 & & 1.0 & & 1.0 & & \\
\hline \multicolumn{9}{|l|}{ T Stage } \\
\hline $\mathrm{T} 1$ & 10 & 90.0 & & 1.0 & & 1.0 & & \\
\hline $\mathrm{T} 2$ & 27 & 84.5 & 0.243 & 2.91 & 0.318 & 2.71 & $0.31-23.4$ & 0.365 \\
\hline $\mathrm{T} 3-\mathrm{T} 4$ & 23 & 63.5 & & 5.07 & 0.125 & 5.39 & $0.64-45.1$ & 0.120 \\
\hline \multicolumn{9}{|c|}{ Clinical staging nodes } \\
\hline No & 49 & 82.9 & 0.034 & 1.0 & & 1.0 & & \\
\hline $\mathrm{N}+$ & 11 & 51.9 & & 3.00 & 0.031 & 3.29 & $1.08-9.99$ & 0.036 \\
\hline \multicolumn{9}{|l|}{ G } \\
\hline G1-G2 & 7 & 83.3 & & 1.0 & & 1.0 & & \\
\hline G3 & 30 & 75.3 & 0.798 & 1.98 & 0.516 & 1.61 & $0.19-13.72$ & 0.663 \\
\hline Missing & 23 & 77.8 & & 1.77 & 0.596 & 1.37 & $0.15-12.14$ & 0.779 \\
\hline \multicolumn{9}{|l|}{ ER status } \\
\hline Negative & 40 & 74.4 & 0.417 & 1.0 & & 1.0 & & \\
\hline Positive & 20 & 83.3 & & 0.63 & 0.419 & 0.14 & $0.02-0.99$ & 0.049 \\
\hline
\end{tabular}


Table IV. Continued.

$\mathrm{B}, \mathrm{BRCAl}$ carriers

\begin{tabular}{|c|c|c|c|c|c|c|c|c|}
\hline \multirow[b]{2}{*}{ Factor } & \multirow[b]{2}{*}{$\mathrm{N}$} & \multirow{2}{*}{$\begin{array}{c}5 \text {-year survival } \\
\text { rate }\end{array}$} & \multirow{2}{*}{$\begin{array}{l}\text { Test log rank } \\
\text { P-value }\end{array}$} & \multicolumn{2}{|c|}{ Univariate } & \multicolumn{3}{|c|}{ Multivariate } \\
\hline & & & & HR & P-value & HR & $95 \%$ CI & P-value \\
\hline \multicolumn{9}{|c|}{ Triple negative } \\
\hline No & 23 & 81.3 & & 1.0 & & 1.0 & & \\
\hline Yes & 37 & 75.1 & 0.884 & 1.08 & 0.883 & 0.20 & $0.03-1.17$ & 0.073 \\
\hline
\end{tabular}

HR, hazard ratio; CI, confidence interval; T, tumor size; N, node; G, grade; ER, estrogen receptor.

$B R C A$ mutation carriers had a significantly worse survival rate compared with non-carriers $(\mathrm{P}=0.017)$ (Fig. 9). The ten-year OS rate was $78.0 \%$ for all analyzed groups: $65.9 \%$ for $B R C A$ mutation carriers and $81.1 \%$ for non-carriers. The 5-year (OS) rate was $86.2 \%$ for all analyzed groups: $77.3 \%$ for $B R C A$ mutation carriers and $88.1 \%$ for non-carriers. In univariate analyses, $B R C A$ mutation carriers had a significantly higher risk of mortality in comparison to non-carriers $(\mathrm{HR}=1.87,95 \% \mathrm{CI}, 1.08-3.25)$ (Table V). After adjusting for other prognostic factors, there was a significant difference in survival between carriers and non-carriers $(\mathrm{HR}=2.28$, $\mathrm{P}=0.019)$. Higher tumor grade $(\mathrm{T} 3-4)(\mathrm{HR}=3.64)$, lymph node metastases $(\mathrm{N}+)(\mathrm{HR}=2.45)$ and $\mathrm{G} 3(\mathrm{HR}=2.84)$ were significant factors for a worse OS. ER+ status was associated with a better $\mathrm{OS}(\mathrm{HR}=0.49, \mathrm{P}=0.022)$. Younger age $(\leq 40$ years $)(\mathrm{HR}=0.48$, $\mathrm{P}=0.081$ ) was a favorable factor, but was not significant. Detailed results for multivariate analysis are shown in Table V.

\section{Discussion}

In this retrospective study, we reported the negative factors for $\mathrm{OS}$ in breast cancer patients with $B R C A$ mutation which were: Infiltration of armpit lymph nodes $(\mathrm{P}=0.034)$, increased size of primary tumor $(\mathrm{T} 3-\mathrm{T} 4, \mathrm{P}=0.243)$, age $>40$ years $(\mathrm{P}=0.310)$ and negative steroid receptor status $(\mathrm{P}=0.417)$. In case of non-carriers, negative factors for OS were also: Lymph node metastasis $(\mathrm{N}+)$ $(\mathrm{P}=0.0008)$, increased tumor size (T3-T4) $(\mathrm{P}=0.0001)$, negative steroid receptor status $(\mathrm{P}=0.054)$ and HER2 overexpression, however this was not significant $(\mathrm{P}=0.273)$.

In a previous study involving a group of patients with stage I breast cancer, $B R C A$ mutation carriers, the ten-year survival rate was $89.9 \%$. Huzarski et al (9) reported that the ten-year OS among breast cancer patients with BRCAl mutation is similar to OS in women without a $B R C A 1$ mutation. Similarly, survival outcomes of $B R C A 1$ mutation carriers were similar to those of sporadic breast cancer patients in a study conducted by Goodwin et al (10). Worse survival outcomes in BRCA2 mutation carriers were observed in univariable analysis (more adverse tumor characteristics). However, similar outcomes of $B R C A 2$ mutation carriers and sporadic disease were identified in multivariable analyses (10). In previous reports, breast cancer $B R C A$ mutation carriers exhibited a worse prognosis compared with breast cancer patients of the same age that did not have the BRCA mutation $(11,12)$. In our study, the ten-year

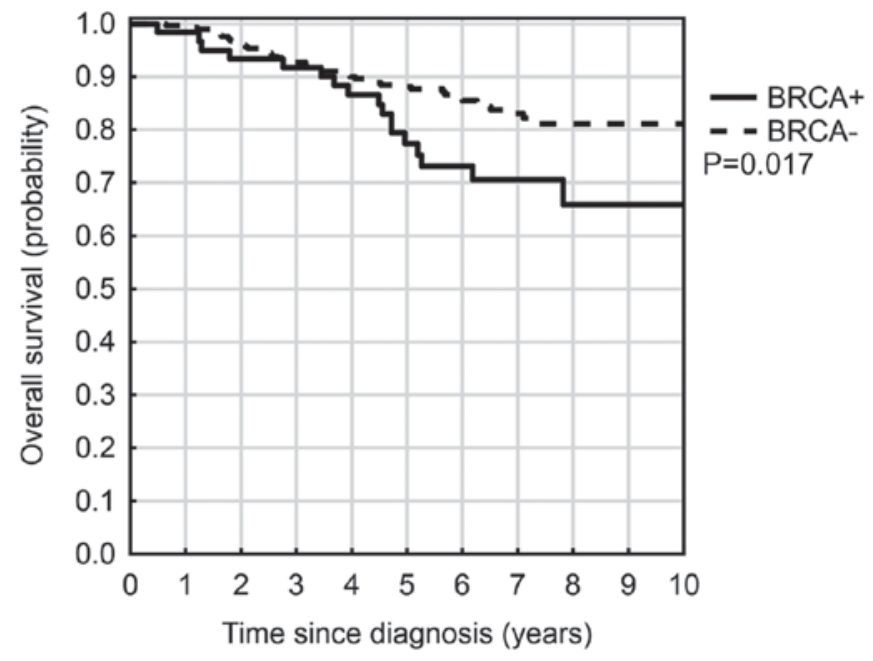

Figure 9. Overall survival analysis according to the presence of BRCA mutation. $\mathrm{P}=0.017$.

OS rate was $65.9 \%$ for BRCA mutation carriers and $81.1 \%$ for non-carriers, irrespective of disease stage. Lee et al (4) showed that the presence of $B R C A 1$ mutation decreases short-term and long-term OS rate, and short-term progression-free survival rate (PFSR). Conversely, there was no reported association between $B R C A 2$ mutation and short-term or long-term survival rate. This suggests that carcinogenic pathways for $B R C A 1$ and BRCA2 are different (13). Baretta et al (14) revealed that patients with $B R C A 1$ mutation have worse OS in comparison to $B R C A$-sporadic patients (HR $1.30 ; 95 \% \mathrm{CI}, 1.11-1.52$ ). Similarly, worse breast cancer-specific survival was reported in BRCAl mutation carriers among patients with stage I-III breast cancer (HR, 1.45; 95\% CI, 1.01-2.07) (14). The meta-analyses conducted by Van der Broek et al (15) did not support worse survival in breast cancer for patients with BRCA1/2 mutation in the adjuvant treatment. They only improved a $10 \%$ worse unadjusted recurrence-free survival for $B R C A 1$ mutation carriers (15). In the present study, $B R C A$ mutation carriers had a significantly worse survival rate compared with non-carriers $(\mathrm{P}=0.017)$. However, patients with the $B R C A$ mutation had an increased rate of TNBC diagnosis in comparison to those with sporadic breast cancer $(61.7 \%$ vs. $15.0 \%, \mathrm{P}=0.0001)$.

Clinicopathological factors affecting OS were also analyzed in various studies. The survival rate for $B R C A$ 
Table V. Multivariate analysis for overall survival.

\begin{tabular}{lrr}
\hline Factor & Hazard ratio & P5\% CI \\
\hline BRCA mutation carriers vs. non carriers (univariable) & 1.87 & 0.026 \\
BRCA mutation carriers vs. non carriers (adjusted) & 2.28 & $1.08-3.25$ \\
Adjusted for: & & $1.15-4.55$ \\
Age (years) & 0.48 & 0.019 \\
T2 vs. T1 & 2.33 & $0.21-1.10$ \\
T3-4 vs. T1 & 3.64 & $1.07-5.08$ \\
N+ vs. N0 & 2.45 & $1.61-8.20$ \\
G3 vs. G1-2 & 2.84 & $1.45-4.14$ \\
G missing vs. G1-2 & 2.82 & 0.002 \\
ER positive vs. ER negative & 0.49 & 0.001 \\
TNBC vs. others & 0.61 & 0.012 \\
\hline
\end{tabular}

T, tumor size; N, node; G, histological grade; ER, estrogen receptor; TNBC, triple negative breast cancer; CI, confidence interval.

positive women without lymph node infiltration and tumor size $<1 \mathrm{~cm}$ was not increased, compared with patients with tumor size between 1 and $2 \mathrm{~cm}$ (10). In the present study, the risk of mortality depended on the stage of the disease and was higher at the advanced T3-T4 stages in BRCA mutation non-carriers and in patients with the $B R C A$ mutation. Huzarski et al (9) reported that oophorectomy significantly improved survival among women with a BRCAl mutation. BRCAl mutation carriers who received chemotherapy had better survival in comparison to women treated without chemotherapy (9). In the Goodwin et al (10) study, the survival of BRCAl mutation carriers treated with chemotherapy was similar to that of $B R C A 1$ non-carriers. However, in case of treatment without chemotherapy, the survival of $B R C A 1$ mutation carriers was worse $(\mathrm{HR}=1.97$; 95\% CI, 0.65-5.94) (10). In our study, all patients received chemotherapy; $97.3 \%$ of patients received chemotherapy regimens with anthracycline.

Foulkes et al (11) confirmed that BRCAl mutation carrier status was associated with clinicopathological factors of breast cancer associated with worse prognosis, including young age at diagnosis, high nuclear grade, negative steroid receptor status (ER-), and the presence of somatic TP53 mutations. In the group of patients with negative steroid receptor status (ER-) tumors, higher nuclear grade 3 and tumor size $<20 \mathrm{~mm}$ the $B R C A 1$ positive status was associated with a significantly worse prognosis (11). Previous studies have confirmed these results $(7,16,17)$. Osin and Lakhani reported that $B R C A 1$-associated tumors are more likely to be steroid receptor negative, and more frequently express p53 protein. Mutations in the TP53 gene also appear to be increased in tumors with BRCA1 mutation (18). The presence of steroid receptor status (ER) in tumors with $B R C A 1$ mutation was significantly lower (8 vs. 26\%) in comparison with a grade-matched control group. In contrast, the presence of ER in tumors with $B R C A 2$ mutation appears to be similar to that in sporadic breast cancers $(13,19)$. In some studies, there was no difference between mutation carriers and non-carriers according to HER2/neu overexpression or amplification $(17,20)$. Crook et al (20) showed that tumors with $B R C A$ mutation were more often p53 positive in comparison to sporadic breast cancers (77\% BRCA1, $45 \%$ BRCA2, $35 \%$ sporadic). The presence of mutations in the TP53 gene have also been reported to be increased in BRCA1 tumors (18). In our analysis, negative prognostic factors for both groups (BRCA mutation carriers and non-carriers) were lymph node metastases, negative steroid receptor status and larger tumor size.

$B R C A$ mutation carriers were characterized by younger age, negative steroid receptor status, tumors without HER2 overexpression and larger tumor size (T3-T4). The ten-year survival rate among breast cancer patients with the BRCAI mutation was significantly worse than in patients without a $B R C A 1$ mutation. Negative factors for OS in breast cancer patients who were carriers of $B R C A$ mutations included infiltration of armpit lymph nodes, negative steroid receptor status and increased size of the primary tumor.

\section{Acknowledgements}

The authors would like to thank Dr Karolina Tęcza, Dr Jolanta Pamuła Piłat and Magdalena Mazur from the Center for Translational Research and Molecular Biology of Cancer for their assistance.

\section{Funding}

No funding was received.

\section{Availability of data and materials}

All data generated or analyzed during this study are included in this published article.

\section{Authors' contributions}

$\mathrm{JH}$ analyzed and interpreted the patient data and was a major contributor in writing the manuscript. ZK performed statistical analysis, and analyzed and interpreted the patient data. 
EG made substantial contributions to conception and design, or acquisition of data, or analysis and interpretation of data. All authors read and approved the final manuscript.

\section{Ethics approval and consent to participate}

All patients provided written informed consent allowing for their biological material to be used in clinical research.

\section{Patient consent for publication}

Not applicable.

\section{Competing interests}

The authors declare that they have no competing interests.

\section{References}

1. Balmaña J, Díez O, Rubio LT and Cardoso F; ESMO Guidelines Working Group: BRCA in breast cancer: ESMO clinical practice guidelines. Ann Oncol 22 (Suppl 6): vi31-vi34, 2011.

2. Andrieu N, Goldgar DE, Easton DF, Rookus M, Brohet R, Antoniou AC, Peock S, Evans G, Eccles D, Douglas F, et al: Pregnancies, breast-feeding, and breast cancer risk in the International BRCA1/2 Carrier Cohort Study (IBCCS). J Natl Cancer Inst 98: 535-544, 2006

3. Ritte R, Tikk K, Lukanova A, Tjønneland A, Olsen A, Overvad K, Dossus L, Fournier A, Clavel-Chapelon F, Grote V, et al: Reproductive factors and risk of hormone receptor positive and negative breast cancer: A cohort study. BMC Cancer 13: 584, 2013.

4. Lee EH, Park SK, Park B, Kim SW, Lee MH, Ahn SH, Son BH, Yoo KY and Kang D; KOHBRA Research Group; Korean Breast Cancer Society: Effect of BRCA1/2 mutation on short-term and long-term breast cancer survival: A systematic review and meta-analysis. Breast Cancer Res Treat 122: 11-25, 2010.

5. Peshkin BN, Alabek ML and Isaacs C: BRCA1/2 mutations and triple negative breast cancer. Breast Dis 32: 25-33, 2010.

6. Bayraktar S, Gutierrez-Barrera AM, Liu D, Tasbas T, Akar U, Litton JK, Lin E, Albarracin CT, Meric-Bernstam F, Gonzalez-Angulo AM, et al: Outcome of triple-negative breast cancer in patients with or without deleterious BRCA mutations. Breast Cancer Res Treat 130: 145-153, 2011.

7. Rennert G, Bisland-Naggan S, Barnett-Griness O, Bar-Joseph N, Zhang S, Rennert HS and Narod SA: Clinical outcomes of breast cancer in carriers of BRCA1 and BRCA2 mutations. N Engl J Med 357: 115-123, 2007.

8. Górski B, Byrski T, Huzarski T, Jakubowska A, Menkiszak J, Gronwald J, Pluzańska A, Bebenek M, Fischer-Maliszewska L, Grzybowska E, et al: Founder mutations in the BRCA1 gene in Polish families with breast-ovarian cancer. Am J Hum Genet 66 : 1963-1968, 2000.
9. Huzarski T, Byrski T, Gronwald J, Górski B, Domagala P, Cybulski C, Oszurek O, Szwiec M, Gugala K, Stawicka M, et al: Ten-year survival in patients with BRCA1-negative and BRCA1-positive breast cancer. J Clin Oncol 31: 3191-3196, 2013.

10. Goodwin PJ, Phillips KA, West DW, Ennis M, Hopper JL, John EM, O'Malley FP, Milne RL, Andrulis IL, Friedlander ML, et al: Breast cancer prognosis in BRCA1 and BRCA2 mutation carriers: An International Prospective Breast Cancer Family Registry population-based cohort study. J Clin Oncol 30: 19-26, 2012.

11. Foulkes WD, Chappuis PO, Wong N, Brunet JS, Vesprini D, Rozen F, Yuan ZQ, Pollak MN, Kuperstein G, Narod SA and Bégin LR: Primary node negative breast cancer in BRCA1 mutation carriers has a poor outcome. Ann Oncol 11: 307-313, 2000.

12. Stoppa-Lyonnet D, Ansquer Y, Dreyfus H, Gautier C, Gauthier-Villars M, Bourstyn E, Clough KB, Magdelénat H, Pouillart P, Vincent-Salomon A, et al: Familial invasive breast cancers: Worse outcome related to BRCA1 mutations. J Clin Oncol 18: 4053-4059, 2000

13. Osin P, Gusterson BA, Philp E, Waller J, Bartek J, Peto J and Crook T: Predicted anti-oestrogen resistance in BRCA-associated familial breast cancers. Eur J Cancer 34: 1683-1686, 1998.

14. Baretta Z, Mocellin S, Goldin E, Olopade OI and Huo D: Effect of BRCA germline mutations on breast cancer prognosis: A systematic review and meta-analysis. Medicine (Baltimore) 95: e4975, 2016.

15. Van der Broek AJ, Schmidt MK, van't Veer LJ, Tollenaar RA and van Leeuwen FE: Worse breast cancer prognosis of BRCA1/BRCA2 mutation carriers: What's the evidence? A systematic review with meta-analysis. PLoS One 10: e0120189, 2015.

16. Robson M, Rajan P, Rosen PP, Gilewski T, Hirschaut Y, Pressman P, Haas B, Norton L and Offit K: BRCA-associated breast cancer: Absence of a characteristic immunophenotype. Cancer Res 58: 1839-1842, 1998.

17. Eisinger F, Stoppa-Lyonnet D, Longy M, Kerangueven F, Noguchi T, Bailly C, Vincent-Salomon A, Jacquemier J, Birnbaum D and Sobol H: Germ line mutation at BRCA1 affects the histoprognostic grade in hereditary breast cancer. Cancer Res 56: 471-474, 1996.

18. Osin PP and Lakhani SR: The pathology of familial breast cancer: Immunohistochemistry and molecular analysis. Breast Cancer Res 1: 36-40, 1999.

19. Armes JE, Trute L, White D, Southey MC, Hammet F, Tesoriero A, Hutchins AM, Dite GS, McCredie MR, Giles GG, et al: Distinct molecular pathogeneses of early-onset breast cancers in BRCA1 and BRCA2 mutation carriers: A population-based study. Cancer Res 59: 2011-2017, 1999.

20. Crook T, Brooks LA, Crossland S, Osin P, Barker KT, Waller J, Philp E, Smith PD, Yulug I, Peto J, et al: p53 mutation with frequent novel condons but not a mutator phenotype in BRCA1-and BRCA2-associated breast tumors. Oncogene 17: 1681-1689, 1998. 